\title{
Optimal Image Quality Assessment based on Distortion Classification and Color Perception
}

\author{
Jee-Yong Lee and Young-Jin Kim \\ Department of Electrical and Computer Engineering, Ajou University \\ San 5, Woncheon-dong, Yeongtong-gu, Suwon 443-749, Republic of Korea \\ [e-mail: neo9912@ajou.ac.kr, youngkim@ajou.ac.kr] \\ *Corresponding author: Young-Jin Kim \\ Received July 19, 2015; revised October 12, 2015; accepted October 25, 2015; \\ published January 31, 2016
}

\begin{abstract}
The Structural SIMilarity (SSIM) index is one of the most widely-used methods for perceptual image quality assessment (IQA). It is based on the principle that the human visual system (HVS) is sensitive to the overall structure of an image. However, it has been reported that indices predicted by SSIM tend to be biased depending on the type of distortion, which increases the deviation from the main regression curve. Consequently, SSIM can result in serious performance degradation. In this study, we investigate the aforementioned phenomenon from a new perspective and review a constant that plays a big role within the SSIM metric but has been overlooked thus far. Through an experimental study on the influence of this constant in evaluating images with SSIM, we are able to propose a new solution that resolves this issue. In the proposed IQA method, we first design a system to classify different types of distortion, and then match an optimal constant to each type. In addition, we supplement the proposed method by adding color perception-based structural information. For a comprehensive assessment, we compare the proposed method with 15 existing IQA methods. The experimental results show that the proposed method is more consistent with the HVS than the other methods.
\end{abstract}

Keywords: image quality assessment, human visual system, structural similarity index, distortion classification, color perception

This work was supported by the National Research Foundation of Korea (NRF) grant funded by the Korea government (MSIP) (No. 2015R1A2A2A01008434). 


\section{Introduction}

There has been substantial growth in the area of multimedia technologies and applications, with a considerable amount of the generated content composed of images. Unfortunately, however, the visual quality of an image is often degraded during its acquisition and subsequent compression, transmission, processing, and reproduction. Therefore, assessing the quality of an image in a human visual system (HVS)-aware manner by measuring the degree of degradation has become crucial in the field of image processing.

There are two categories of image quality assessment (IQA) methods: subjective and objective [1]. Subjective methods require quality assessment by the human eye, which is usually inconvenient, time-consuming, and expensive. In contrast, objective methods use mathematical models that can automatically predict the image quality. The peak signal to noise ratio (PSNR) and mean squared error (MSE) are the best-known objective metrics, largely a result of their low complexity and clear physical meaning. However, they show poor correlation with the HVS $[2,3]$.

In order to overcome these disadvantages, many researchers have investigated how best to evaluate image quality in an HVS-aware manner. To this end, Structural SIMilarity (SSIM) [4] is proposed to reflect that the HVS is highly adapted to extract structural information from a visual scene. Because SSIM measures quality degradation as a perceived change in structural information, its experimental results indicate a higher correlation with the HVS than PSNR and MSE.

However, it has been reported that SSIM has some critical problems concerning external and internal factors. As SSIM does not consider which type of degradation affects an image's structure, a predicted index by SSIM tends to be biased according to the type of distortion [5]. Hence, a single predicted index covers a wide range of subjective scores, which makes it difficult to compare the quality of images with different distortion types. Thus, IQA methods need to compute indices that are perceptually relevant to humans for many types of distortion. Moreover, SSIM also fails to measure the color difference between two images, because it only evaluates the quality of grayscale images and does not make use of any color information [6]. According to [7], color vision is a critical component of human vision, and plays an important role in perception. Moreover, we are now exposed to a large number of color images through television, computers, smartphones, and other devices in which color plays a significant role. Therefore, color information should be considered in quality assessment.

In this paper, to overcome these problems, we first design a classification system based on an investigation into the features of distorted images. The classification system categorizes different distortion types and matches an optimal constant with a specific type of distortion. This constant is a crucial parameter constituting an IQA metric. The IQA metric can then compute the numerical quality of an image using these constants according to the distortion type. In addition, we propose an improved HVS-aware objective IQA metric by considering the perceptual color difference between two color images. Through extensive experiments, we show that the proposed method, which combines a classification system and an improved metric, has a better correlation with the HVS than 15 existing IQA methods.

The remainder of this paper is organized as follows. In Section 2, we present an overview of SSIM and its two significant limitations. Section 3 explains how the bias problem can be solved and discusses the proposed metric based on perceptual color differences in detail. Section 4 presents and discusses the experimental results. Finally, Section 5 contains our 
conclusions.

\section{Related Work}

SSIM is a full reference (FR) IQA metric. The principal idea underlying SSIM, proposed by Wang [4], is that the HVS is highly adaptable for the extraction of structural information from visual scenes. SSIM consists of three parts: luminance similarity, contrast similarity, and structure correlation between two signals $x$ and $y$ (see Table 1).

Table 1. Three parts constituting SSIM and the definition of SSIM

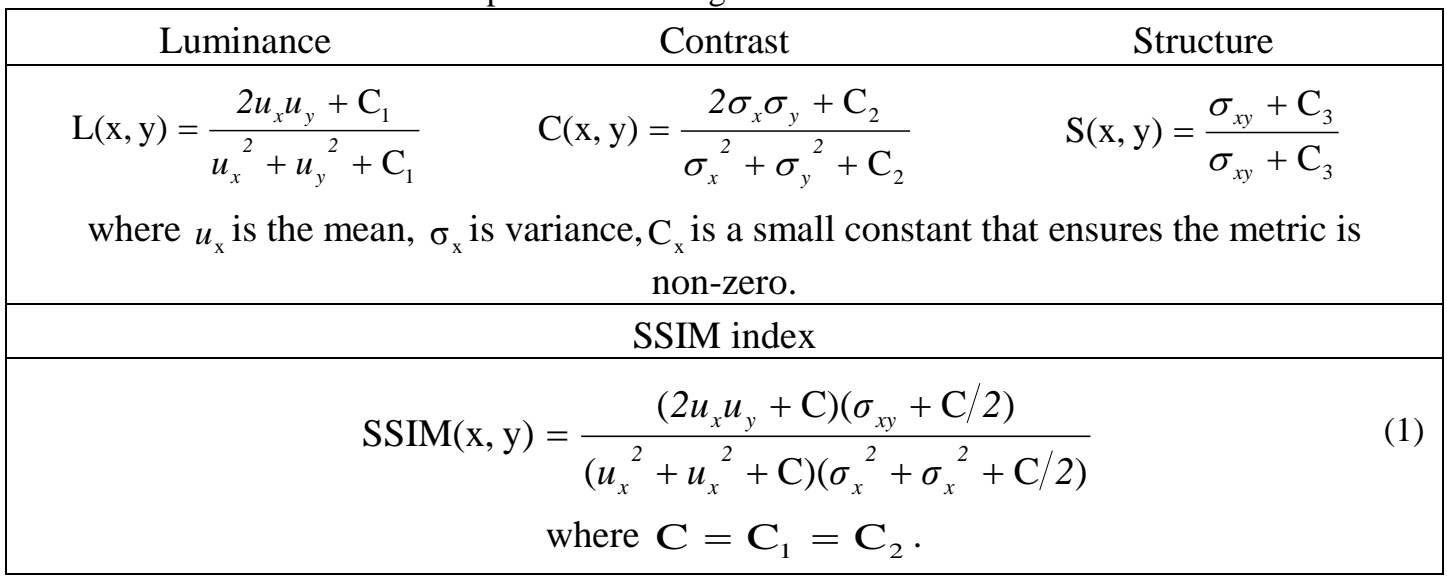

The SSIM index is calculated on a local window that moves pixel-by-pixel across the whole image space. The overall quality of an image (i.e., the final SSIM index) can be obtained as the mean of the SSIM indices calculated on all local windows, which is shown in Eq. (2).

$$
\operatorname{Quality}(\mathrm{X}, \mathrm{Y})=\frac{1}{M} \sum_{j=1}^{M} \operatorname{SSIM}\left(\mathrm{x}_{j}, \mathrm{y}_{j}\right)
$$

where $\mathrm{X}$ and $\mathrm{Y}$ are the original and distorted images, respectively, $x$ and $y$ are the image contents in the $j$-th local window, and $M$ is the number of local windows in the image. The value of SSIM ranges from 0 to 1 , with higher values indicating more similarity between the images. SSIM is an effective IQA tool because of its solid theory and good performance.

There are many modified versions of SSIM. For example, MSSIM calculates SSIM in a multi-scale way [8], and IW-SSIM [9] includes weighted local information and multi-scaling. Some researchers found that the gradient magnitude may capture the features of the HVS. GSM [10] directly incorporates this finding into IQA metrics. FSIM [11] employs phase congruency as features, respectively, to compare the structural difference. The above techniques have lead to some improvement in the accuracy of evaluation, but often result in poor performance in the presence of noise types such as blur, and White noise..

Meanwhile, pattern recognition paradigms allow to tackle the consideration of structural information work from a different perspective, as the eventual goal is to mimic quality perception by using a variety of visual features. Several studies $[12,13]$ have demonstrated the ability of pattern recognition-based approaches to consider structural information. 


\section{Motivation}

It is generally recognized that SSIM has two critical problems. Fig. 1 (a) shows the scatter plots of SSIM indices (x-axis) versus subjective scores (y-axis) for images from the LIVE database [14]. Subjective scores input into the database were obtained from about two dozen human subjects. A lower subjective score indicates that the image provides better perceptual quality. The red line is a regression curve between the two variables. In this graph, the SSIM indices seem to have a good relation with the subjective scores, but there are still many outliers around the regression curve. These make it difficult to compare the quality of images with different types of distortion. In Fig. 1 (b), we can see that the blue and red points representing White noise and JPEG images, respectively are distinctly differentiated from an the other three types of distortions. Therefore, if it were possible to classify these images correctly, we would be able to obtain optimal results through an efficient classification-based evaluation.

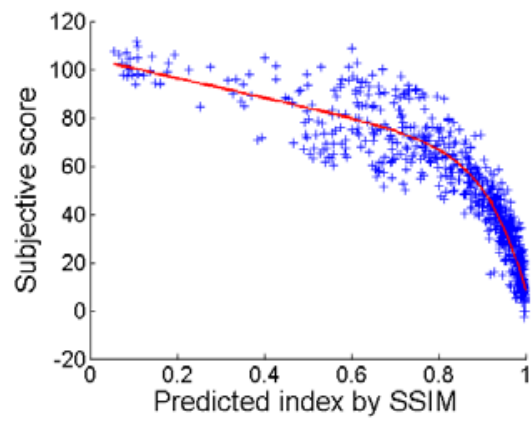

(a)

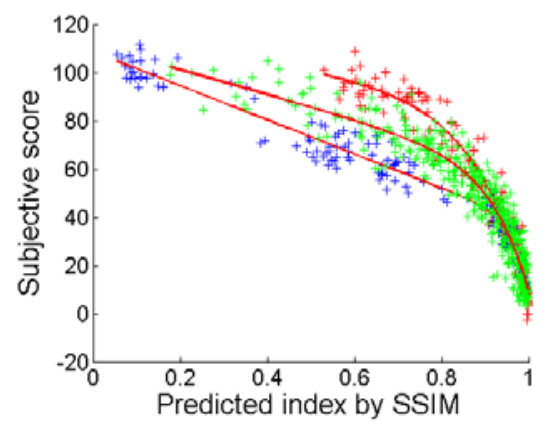

(b)

R: JPEG, B: White Noise, G: Others

Fig. 1. Scatter plots of subjective scores versus SSIM indices on the LIVE database.
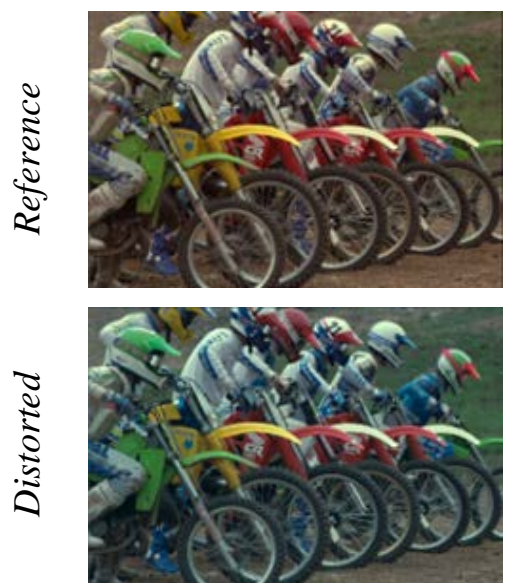

(a) Bike from LIVE database $($ SSIM $=0.9911)$
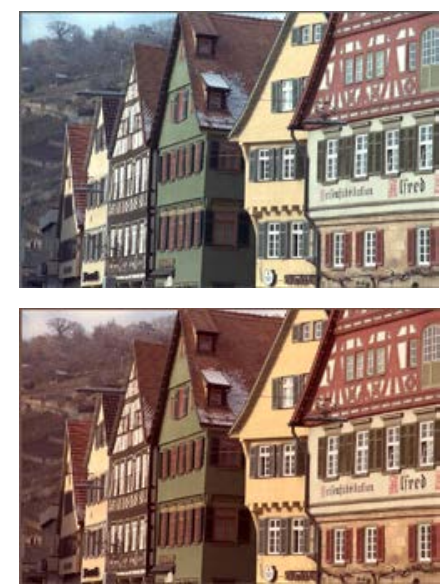

(b) Buildings from LIVE database $($ SSIM $=0.9967)$
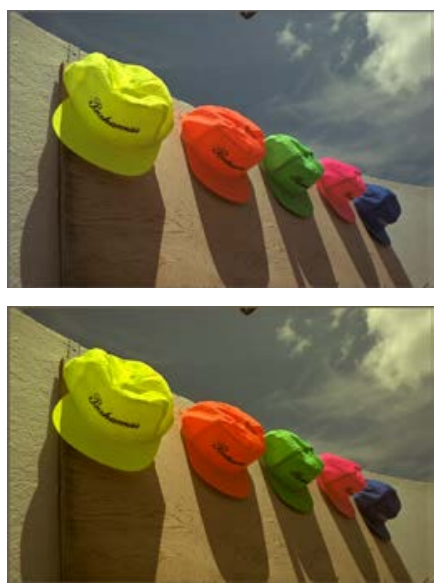

(c) Caps from LIVE database $($ SSIM $=0.9984)$

Fig. 2. Original images and color-distorted images with SSIM scores 
Meanwhile, Fig.2 illustrates the color-blindness problem SSIM has. As SSIM only evaluates the quality of an image after transforming to grayscale image, it cannot make use of any color information. In Fig. 2, first row shows three images from the LIVE database. The second row shows the corresponding color-distorted images. The SSIM indices of these three pairs (i.e., (a), (b) and (c)) are 0.9911, 0.9967, and 0.9984, respectively. They are all higher than 0.9900, which means that SSIM judges the distorted images to be almost identical to the originals. However, it is clear that their color tones are quite different in terms of human visual perception. Therefore, this color-blindness problem of SSIM requires improvement.

\section{Proposed Method}

\subsection{Classification system}

\subsubsection{Feature description of the image}

In this paper, we propose a new IQA method that considers different indices according to the types of distortion and color information contained within an image. We first design a classification system for matching an optimal constant to the type of distortion. Then, we improve the method by considering visual color perception. The implementation of our method is explained as follows.

$$
\mathrm{E}(i, j)=-\sum_{x=i-4}^{i+4} \sum_{y=j-4}^{j+4} \mathrm{G}(x, y) \log _{2} \mathrm{G}(x, y)
$$

To obtain a pixel value of E, the 9-by-9 neighborhood around the pixel of interest in image G is used, where $(\mathrm{i}, \mathrm{j}$ ) are the pixel coordinates of E. For pixels on the borders of E, symmetric padding is used. In symmetric padding, the values of the padding pixels are the mirror reflection of the border pixels in $\mathrm{G}$.

As a secondary feature, phase congruency (PC) is exploited for classification. PC is the local energy model developed by Morrone et al. [15]. It postulates that features are perceived at the points where the Fourier components attain their maximal phase. Among the various implementations for computing the PC map of a given image, we adopt that of Kevesi [16], which is widely used in the literature. To obtain the local frequency information, particularly the local phase, banks of filters are required in a quadrature tuned to different spatial frequencies. In this study, log-Gabor filters are used [17]. Let $\mathrm{G}$ denote the grayscale image of the input image, $\mathrm{M}_{\mathrm{s}, \mathrm{o}}^{\text {even }}$ and $\mathrm{M}_{\mathrm{s}, \mathrm{o}}^{\text {odd }}$ denote the even-symmetric and odd-symmetric filters at scale $s$ and orientation $o$, respectively. The response vector can then be obtained from the response of the quadrature filter pair: $\left[e_{s, 0}(i, j), o_{s, o}(i, j)\right]=\left[G(i, j) * M_{s, o}^{\text {even }}, G(i, j) * M_{s, 0}^{\text {odd }}\right]$. The amplitude of the response and the phase are given by $\mathrm{A}_{s, o}(i, j)=\sqrt{e_{s, o}(i, j)^{2}+o_{s, o}(i, j)^{2}}$ and the local energy along the orientation $o$ is $\mathrm{L}_{o}(i, j)=\sqrt{\mathrm{F}_{o}(i, j)^{2}+\mathrm{H}_{o}(i, j)^{2}}$, where $\mathrm{F}_{o}(i, j)=\sum_{s} e_{s, o}(i, j)$ and $\mathrm{H}_{o}(i, j)=\sum_{s} o_{s, o}(i, j)$. The 2D PC coordinates $(i, j)$ is defined as:

$$
\mathrm{PC}_{o}(i, j)=\frac{\mathrm{L}_{o}(i, j)}{\varepsilon+\sum_{s} \mathrm{~A}_{s, o}(i, j)}
$$




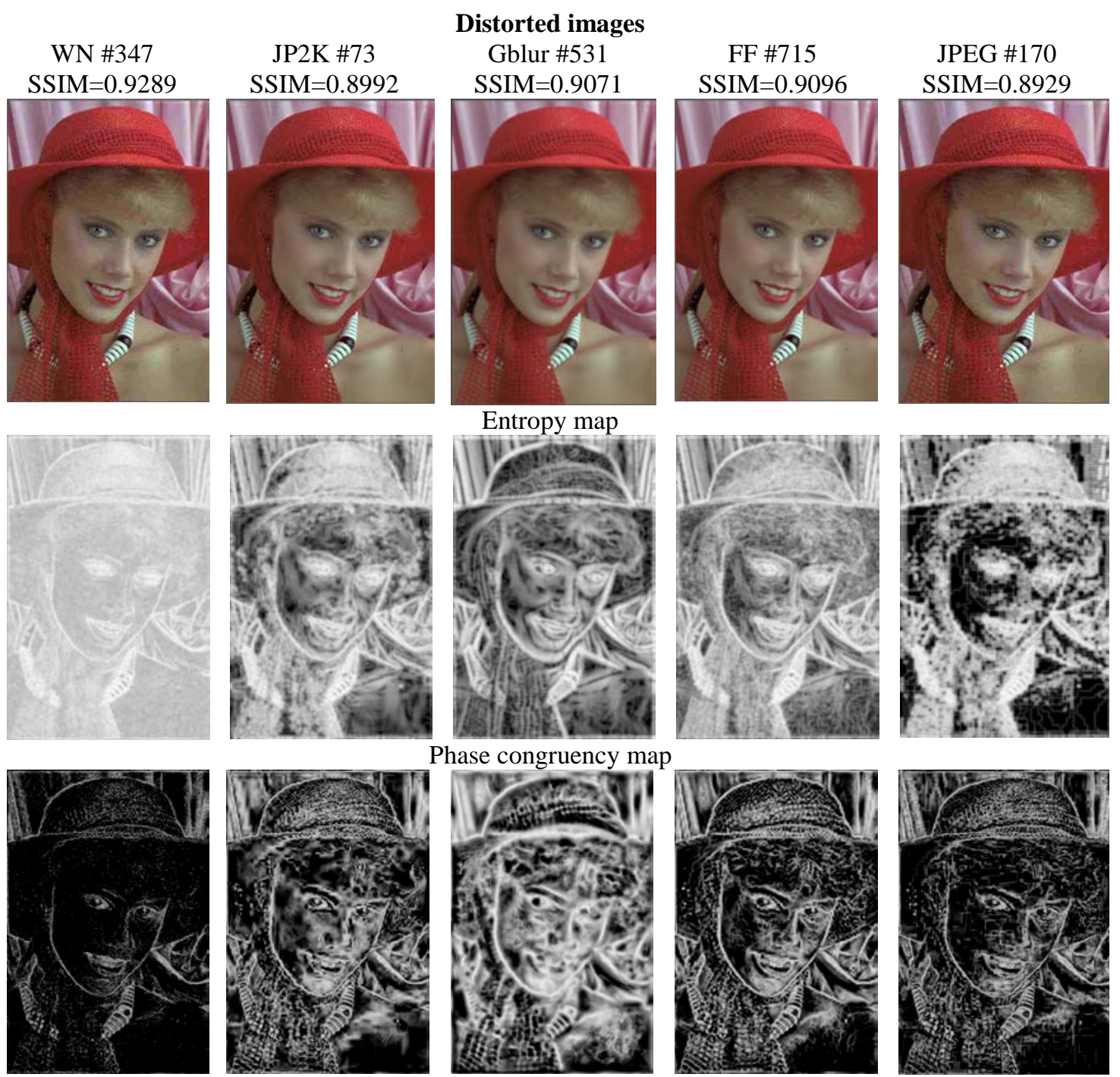

Fig. 3. Sensitivity example of the computed features of the 'woman' image with different distortion types, which is taken from the LIVE database.

Note that PC $(i, j)$ returns a real number in the range [0 1]. We use a MATLAB program for computing the PC map, which is implemented and released in [11].

Fig. 3 exemplifies the sensitivity of each feature map (second and third rows) according to the distortion types on the 'woman' image from the LIVE database. Each feature maps are reported for five images that are degraded with different types of distortion while they all have SSIM values of around 0.9000 . Although all the images appear identical, if you observed those images through the feature map, you would figure out that their differences are noticeable. Specifically, in case of White noise, obvious characteristics are observed in the feature map. The entropy map looks brighter than the others and the phase congruency looks darker than the other maps. Further, as there is no considerable White noise, feature maps of JPEG are also noticeably different.

By using statistical percentiles for these feature maps, we can represent the distribution of each feature map. Fig. 4 illustrates how each distribution curve can effectively express each 
distortion's characteristic in the feature maps, where (a) and (b) are graphs about entropy and phase congruency maps, respectively. These graphs show the results of the 'woman' image from the LIVE database for the five different types of distortion. The values of the percentiles (y-axis) within the feature map are plotted for the percentages from 0 to 100 (x-axis). We can notice that the results of the images degraded with White noise and JPEG are surely distinct from others in both graphs. The result of White noise is above or below those of the other types of distortion distinctly in both graphs. JPEG also remains below the other types distinctly in both graphs.



(a)

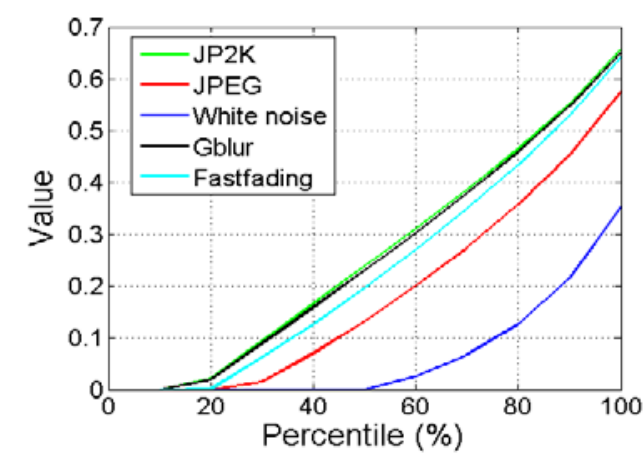

(b)

Fig. 4. Description of distributions of two feature maps of the 'woman' image for five types of distortion.

Similar results are observed for most of the images in the LIVE database. In this paper, to use such results for classification effectively, just 10 values for the percentile from 10 to 100 (\%) with an interval of 10 are extracted from each distribution. Finally, the overall descriptor for classification can be obtained by combining these two vectors.

\subsubsection{Support vector machine for classification}

Support vector machines (SVMs) [18] are a powerful tool for solving binary classification tasks. Given a set, $Z=\left\{\left(x_{l}, y_{l}\right) ; l=1, \ldots, n_{p} ; x_{l} \in \mathrm{X} \subseteq \mathrm{R}^{q} ; y_{l} \in \mathrm{Y}=\{-1,+1\}\right\}$ of $n_{p}$ patterns, and SVM maps the input domain, $\mathrm{X} \subseteq \mathrm{R}^{q}$, into a Hilbert space, $\mathrm{F} \subseteq \mathrm{R}^{m}(q<<m<<\infty)$, where a linear class separation is feasible. SVM relies on the solution of the following quadratic programming problem to find the optimal hyper-plane $w$ separating the two classes:

$$
\min _{\alpha}\left\{\frac{1}{2} \sum_{l, m=1}^{n_{p}} \alpha_{l} \alpha_{m} y_{l} y_{m} \mathrm{~K}\left(x_{l}, x_{m}\right)-\sum_{l=1}^{n_{p}} \alpha_{l}\right\}, \quad \text { subject to }\left(0 \leq \alpha_{l} \leq C, \sum_{l=1}^{n_{p}} y_{l} \alpha_{l}=0\right)
$$

where $\alpha_{l}$ are the SVM parameters setting the class-separating surface and the scalar quantity $\mathrm{C}$ is a fixed regularization term that balances the tradeoff between accuracy and complexity. The problem in (6) has the crucial advantage of involving a quadratic-optimization problem with linear constraints, ensuring that the solution is unique. The kernel function $\mathrm{K}$ supports inner products among patterns in a higher-dimensional transformed space without requiring 
the specific mapping of each pattern. The radial basis function kernel, formulated as $\mathrm{K}\left(x_{1}, x_{2}\right)=\exp \left(-\left\|x_{1}-x_{2}\right\|^{2} / \sigma^{2}\right)$, is a popular and effective choice, and is thus used in the proposed method.

Basically an SVM model inherently solves a two-class problem and thus we should design two models exploiting 'one-versus-the-rest' classifiers for the distortion types of interest (i.e., White Noise, JPEG). Table 2 reports the performance of the designed classification system. It can be seen that the number of misclassified images is very small. In this study, to design an SVM model, we randomly select $80 \%$ of the reference images and their corresponding distorted images for training, and use the remaining $20 \%$ for testing.

Table 2. Confusion matrix for the distortion identifier

\begin{tabular}{|c|c|c|c|c|}
\hline & \multicolumn{3}{|c|}{ Classified } \\
\hline & & White Noise & JPEG & Others \\
\hline \multirow{3}{*}{ 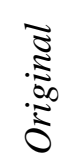 } & \multirow{3}{*}{$\begin{array}{c}\text { White Noise } \\
\text { JPEG } \\
\text { Others }\end{array}$} & 21 & 0 & 0 \\
\hline & & 0 & 22 & 3 \\
\hline & & 1 & 0 & 93 \\
\hline
\end{tabular}

\subsubsection{Operation of the classification system for image quality assessment}

Based on prior investigations, our classification system for optimal quality assessment matches an appropriate constant with the type of distortion. The detailed pseudo-code is shown in Algorithm 1. After the input images have been converted to grayscale image, each feature extraction function obtains two feature vectors from the distorted images. These feature vectors (i.e., descriptors) are then combined to enable classification. The two SVM classifiers for identifying White Noise and JPEG then arrive at a decision (positive or negative with a confidence score). Further, using a distortion decision function, the type of distortion that contained in the image is finally determined. The proposed algorithm operates according to the following rules.

1) If only one classifier gives a positive decision, the image is regarded as having the type of distortion that the classifier identifies.

2) If both classifiers give positive decisions, the distortion type of the image is identified by referring to their confidence scores.

3) If both classifiers give negative decisions, the image is regarded as having one of the 'others' types of distortion.

Based on the results, the image is assigned a constant according to the constant matching table in Table 3, which gives the most appropriate value for computing the relevant image quality under each type of distortion. We obtained these constants by finding the maximum correlation coefficient between the set of subjective scores and the predicted score for images in the LIVE database. This process can be represented as follows:

$$
\max _{C_{W N}, C_{j P E G}}\{\text { correlation (subjective scores, predicted indices) }\}
$$






Table 3. Constant matching table

\begin{tabular}{|l|c|c|c|}
\hline & White Noise & JPEG & Others \\
\hline Constant (C) & 5.12 & 58 & 6.5 \\
\hline
\end{tabular}

Fig. 5 shows various results when (a) the classification system is not applied, and (b) when the classification system for the SSIM metric is applied. The vertical bars in the graph indicate the dispersion of the subjective scores toward a SSIM index value. It can be observed that the points in (b) are gathered more concentrically, where the predicted White noise indices have become larger and those of JPEG have become smaller. Thus, the proposed system enables the metric to compute perceptually relevant indices for many types of distortion. 


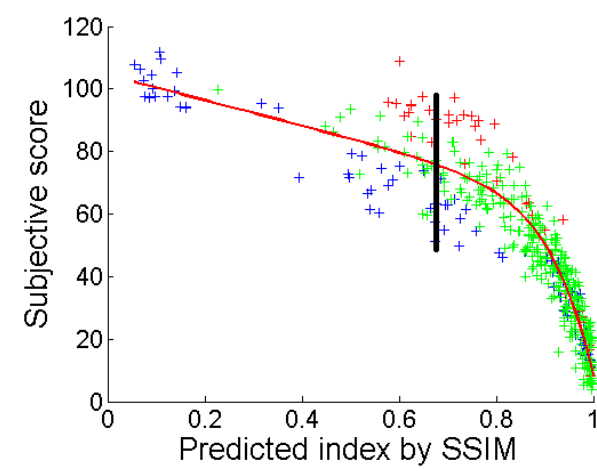

(a)

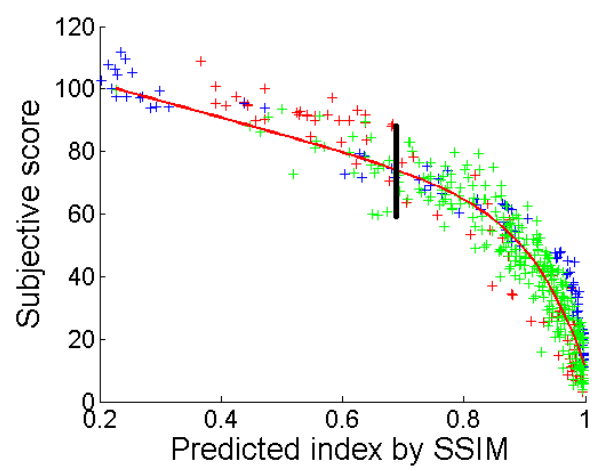

(b)

Fig. 5. Scatter plots of subjective score versus SSIM index on the LIVE database.

\subsection{Perceptual color similarity-based IQA method}

\subsubsection{CIE Lab color space}

To overcome the limitations of SSIM illustrated in Fig. 2, we propose an improved quality assessment metric for color images by reflecting visual color perception. The new metric employs the CIE Lab color space, which is often used in attempts to describe perceptual color differences. It is an approximately uniform color space described by components $L, a$, and $b$. The $L$ component represents the lightness value, whereas $a$ and $b$ represent the values from green to red, and from blue to yellow, respectively. The CIE Lab color space was designed to approximate human vision.

In this color space, perceptual color differences are represented by spatial distances. That is, the perceptual difference between any two colors can be approximated by the Euclidean distance between them, where a color is represented as a vector point with three components in three-dimensional space [19]. In this color space, a uniform change in components corresponds to a uniform change in the perceived color. Thus, assessing perceptual color differences quantitatively can be greatly simplified by transforming RGB values to points in the CIE Lab color space. By using this color space, we design a new quality assessment method for color images.

\subsubsection{Perceptual color similarity-based IQA method}

The procedure of determining perceptual color similarity is as follows. When the original and distorted images $\mathrm{X}$ and $\mathrm{Y}$ are given in the RGB color space, they are converted to $\mathrm{X}_{\mathrm{Lab}}$ and $\mathrm{Y}_{\text {Lab }}$ in the CIE Lab color space using a transformation equation [20]. Thus, each pixel will be represented as a vector point with three components $(L, a, b)$ in the CIE Lab color space. Next, the Euclidean distance $\Delta \mathrm{D}$ between a pair of representative color points in the local window is calculated to obtain the local color difference. Here, the representative color points are obtained by convoluting the window with a Gaussian distribution. When the distance between two colors decreases, the HVS-awareness is considerably affected by small variations in distance, and when the distance between two colors increases, the HVS-awareness is unaffected, because the difference between images is too big to find any similarity. Therefore, the perceptual color difference between two colors is expressed as: 


$$
f\left(x_{i}, y_{i}\right)=1-\exp \left(-\frac{\Delta \mathrm{D}\left(x_{i}, y_{i}\right)}{\gamma}\right)
$$

where $\gamma$ is a normalization factor. Based on Eq. (8), the proposed color similarity function CS is defined as:

$$
\operatorname{CS}\left(x_{i}, y_{i}\right)=\exp \left(-\frac{\Delta \mathrm{D}\left(x_{i}, y_{i}\right)}{\gamma}\right)
$$

Under Eq. (9), the perceptual color similarity between two color points will be mapped to an index that ranges from 0 to 1 . Finally, by including other structural information, the final IQA method, CS-SSIM, is

$$
\mathrm{CS}-\operatorname{SSIM}(x, y)=\exp \left(-\frac{D_{x y}}{\gamma}\right) \frac{\left(2 \sigma_{x y}+C\right)}{\left(\sigma_{x}^{2}+\sigma_{x}^{2}+C\right)}
$$

Here, we discard the luminance similarity, included in the original SSIM. This is because the perceptual color difference can cover the luminance difference. The overall similarity index between images $\mathrm{X}$ and $\mathrm{Y}$ can then be represented by:

$$
\operatorname{Quality}(\mathrm{X}, \mathrm{Y})=\frac{1}{\mathrm{M}} \sum_{i=1}^{\mathrm{M}} \mathrm{CS}-\operatorname{SSIM}\left(x_{i}, y_{i}\right)
$$

where $M$ is the number of local windows in the image. Table 4 reports that CS-SSIM reflects the HVS better than SSIM, when considering the subjective scores and indices together.

Table 4. Comparison of the predicted index by SSIM and CS-SSIM for the color-distorted images in Fig. 2 with subjective scores.

\begin{tabular}{|c|c|c|c|}
\hline & Bikes & Buildings & Caps \\
\hline Subjective score & 3.15 & 3.65 & 3.4 \\
\hline SSIM & 0.9911 & 0.9967 & 0.9984 \\
\hline CS-SSIM & 0.9597 & 0.9631 & 0.9605 \\
\hline
\end{tabular}

\section{Experimental Results}

We chose four criteria for performance evaluation. The first is the root-mean-squared error (RMSE) between subjective scores and predicted scores by an IQA method after nonlinear regression; which is used to evaluate the prediction accuracy evaluation. Small RMSE values denote better accuracy. The second criterion is the linear correlation coefficient (LCC) after non-linear regression, which measures the strength of the linear relationship between two variables. Large LCC values denote better performance. The nonlinearity chosen for regression is a 5-parameter logistic function [21]: 


$$
\operatorname{Score}(x)=\beta_{1}\left(\frac{1}{2}-\frac{1}{1+\mathrm{e}^{\beta_{2}\left(x-\beta_{3}\right)}}\right)+\beta_{4} x+\beta_{5},
$$

The third and fourth metrics are based on rank-order correlation. Spearman's rank correlation coefficient (SRCC) [22] assesses how well the relationship between two variables can be described using a monotonic function, and Kendall's rank correlation coefficient (KRCC) [23] improves on SRCC by reflecting the strength of the dependence between the variables being compared. We evaluated the performance of the proposed method with these four criteria over the LIVE image database, which consists of 29 reference images and 779 distorted images for five types of distortion: JPEG, JPEG2000, White noise, Gaussian blur, and Fastfading [14].

\subsection{Results and Discussion}

Because the proposed IQA method employs an SVM algorithm for quality assessment, we randomly selected $80 \%$ of the reference images and their corresponding distorted images to train the SVM model, and used the rest for testing. To remove any performance bias, the $80 / 20 \%$ training/testing rate was applied and repeated 100 times. Consequently, the average is regarded as the final result.

Table 5. Performance comparison of IQA methods for four criteria.

\begin{tabular}{|c|c|c|c|c|}
\hline Algo. & SRCC & KRCC & LCC & RMSE \\
\hline PSNR & 0.8756 & 0.6865 & 0.8723 & 13.3597 \\
\hline UQI & 0.8941 & 0.7100 & 0.8987 & 11.9823 \\
\hline NQM & 0.9086 & 0.7413 & 0.9122 & 11.1926 \\
\hline IFC & 0.9259 & 0.7579 & 0.9268 & 10.2643 \\
\hline VSNR & 0.9274 & 0.7616 & 0.9231 & 10.5059 \\
\hline RFSIM & 0.9401 & 0.7816 & 0.9354 & 9.6642 \\
\hline SSIM & 0.9479 & 0.7963 & 0.9449 & 8.9455 \\
\hline MSSIM & 0.9513 & 0.8045 & 0.9489 & 8.6188 \\
\hline GSM & 0.9561 & 0.8150 & 0.9512 & 8.4327 \\
\hline IW-SSIM & 0.9567 & 0.8175 & 0.9522 & 8.3473 \\
\hline SR-SIM & 0.9618 & 0.8299 & 0.9553 & 8.0811 \\
\hline FSIM & 0.9634 & 0.8337 & 0.9597 & 7.6780 \\
\hline VIF & 0.9636 & 0.8282 & 0.9604 & 7.6137 \\
\hline FSIMc & 0.9645 & 0.8363 & 0.9613 & 7.5296 \\
\hline MAD & $\mathbf{0 . 9 6 6 9}$ & $\mathbf{0 . 8 4 2 1}$ & $\mathbf{0 . 9 6 7 5}$ & $\mathbf{6 . 9 0 7 3}$ \\
\hline Proposed & $\underline{\mathbf{0 . 9 6 7 1}}$ & $\underline{\mathbf{0 . 8 5 6 1}}$ & $\underline{\mathbf{0 . 9 6 8 9}}$ & $\underline{\mathbf{6 . 8 8 6 5}}$ \\
\hline
\end{tabular}


For comprehensive assessment, we compared the proposed method with 15 other existing IQA indices including PSNR, UQI [2], NQM [24], IFC [25], VSNR [26], RFSIM [27], SSIM [4], MSSIM [8], GSM [10], IW-SSIM [9], SR-SIM [21], FSIM/FSIMc [11], VIF [28], MAD [29], on the LIVE database and the results are listed in Table 5. The two high ranked metrics are highlighted in boldface and the best metric is underlined.

Prior to our research on IQA, the MAD method has shown the best performance on the LIVE database. However, as can be seen in the table, the proposed method outperformed the other indices for all criteria. Compared to SSIM result, the proposed technique showed a performance improvement of 2\% (SRCC), 7\% (KRCC), 2.6\% (LCC), and 23\% (RMSE), while compared to the second best results (given by the MAD method), our technique achieved a performance improvement of $0.02 \%$ (SRCC), 1.7\% (KRCC), 0.14\% (LCC), and $0.3 \%$ (RMSE). Therefore, it can be concluded that the proposed IQA method achieves more consistent HVS-awareness than other existing IQA methods.

In particular, Fig. 6 shows that the dispersion of subjective scores toward SSIM value is much smaller, which means that the proposed index is more consistent with the HVS. The primary reason for this performance improvement by the proposed method is that it considers the type of distortion and tries to compute perceptually relevant indices for each case. In addition, we have designed a new metric that considers perceptual color information. By combining all such methods, the proposed IQA metric can achieve better correlation with subjective scores than other indices for the LIVE database.



Fig. 6. Scatter plots of subjective score versus predicted index by proposed method on the LIVE database.

\section{Conclusions}

In this paper, we have proposed a new IQA method by designing a classification system that determines the optimal constant according to the specific type of distortion. With this system, we can solve the biasing problem of predicted scores by SSIM according to the type of distortion. Moreover, by introducing similarity based on perceptual color differences, we also proposed a new metric called CS-SSIM. CS-SSIM accounts for the fact that human eyes are sensitive to color information as well as the luminance of an image. Extensive experiments showed that the proposed method outperform 15 other quality assessment methods for HVS-awareness using the LIVE database. In future work, we plan to improve this system to enable the identification and classification of a wide variety of distortion types over other databases. 


\section{References}

[1] K. Thung and R. Paramesran, “A survey of image quality measures,” in Proc. of International Conference for Technical Postgraduates (TECHPOS), pp. 1-4, 2009. Article(CrossRef Link)

[2] Z. Wang and A. C. Bovik, “A universal image quality index,” IEEE Signal Processing Letter, vol. 9, no.3, pp. 81-84, Mar. 2002. Article(CrossRef Link)

[3] Z. Wang and A. C. Bovik, "Mean squared error: love it or leave it?," IEEE Signal Processing Magazine, vol. 26, no.1, pp. 98-117, 2009. Article(CroosRef Link)

[4] Z. Wang and A. C. Bovik, "Image quality assessment: from error visibility to structural similarity," IEEE Transactions on Image Processing, vol. 13, no. 4, pp.600-612, 2004. Article(CroosRef Link)

[5] H. Tang, N. Joshi, and A. Kapoor, "Learning a blind measure of perceptual image quality,” in Proc. of International Computer Vision and Pattern Recognition (CVPR), pp. 305-312, Jun. 2011. Article(CroosRef Link)

[6] Y. Shi, Y. Ding, R. Zhang, and J. Li, "Structure and hue similarity for color image quality assessment," in Proc. of the International Conference on Electronic Computer Technology (ICECT), pp. 329-333, Feb. 2009. Article(CroosRef Link)

[7] M.A. Webster, "Human colour perception and its adaptation," Network: Computation in Neural Systems, vol. 7, no. 4, pp. 587-634, Nov. 1996. Article(CroosRef Link)

[8] Z. Wang, E. P. Simoncelli and A. C. Bovik, "Multi-scale structural similarity for image quality assessment," in Proc. of IEEE Asilomar Conference on Signals, Systems and Computers, pp. 1398-1402, 2003. Article(CroosRef Link)

[9] Z. Wang and Q. Li, "Information content weighting for perceptual image quality assessment," IEEE Transactions on Image Processing, vol. 20, no. 5, pp. 1185-1198, May. 2011. Article(CroosRef Link)

[10] A. Liu, W. Lin and M. Narwaria, "Image quality assessment based on gradient similarity," IEEE Transactions on Image Processing, vol. 21, no. 4, pp. 1500-1512, Apr. 2012. Article(CroosRef Link)

[11] L. Zhang, D. Zhang, X. Mou and D. Zhang, "FSIM: A feature similarity index for image quality assessment,” IEEE Transactions on Image Processing, vol. 20, no. 8, pp. 2378-2386, Aug. 2011. Article(CroosRef Link)

[12] Z. Li, J. Liu, J. Tang, and H. Lu, "Robust structured subspace learning for data representation," IEEE Transactions on Pattern Analysis and Machine Intelligence, Feb. 2015, doi:// 10.1109/TPAMI.2015.2400461. Article(CroosRef Link)

[13] Z. Li, J. Liu, Y. Yang, X. Zhou, and H. Lu, "Clustering-guided sparse structural learning for unsupervised feature selection,” IEEE Transactions on Knowledge and Data Engineering, vol.26, no. 9, pp. 2138-2150, Sep. 2014. Article(CroosRef Link)

[14] L. C. H. R. Sheikh, Z. Wang, and A. C. Bovik, "Live image quality assessment database release 2," 2007. [Online] Available: http://live.ece.utexas.edu/research/quality

[15] M. C. Morrone and D.C. Burr, "Feature detection in human vision: a phase-dependent energy model," in Proc. of the Royal Society of London B, vol. 235, no. 1280, pp. 221-245, Dec. 1988. Article(CroosRef Link)

[16] P. Kovesi, "Image features from phase congruency," Videre: Journal of Computer Vision Research, vol. 1, no. 3, pp. 1-26, 1999.

[17] D. J. Field, "Relations between the statistics of natural images and the response properties of cortical cells,” Journal of the Optical Society of America A, vol. 4, no. 12, pp. 2379-2394, Dec. 1987. Article(CroosRef Link)

[18] C. Cortes, and V. Vapnik, "Support vector networks," Machine learning, vol. 20, no. 3, pp. 273-297, Sep. 1995. Article(CroosRef Link)

[19] G. Wyszecki and W.S. Styles, "Color Science: Concepts and Methods, Quantitative Data and Formulae,” Wiley, New York, 1982. Article(CroosRef Link) 
[20] A. Ford and A. Roberts, “Color space conversions,” Westminster University, London, pp. 1-31, 1998.

[21] H. R. Sheikh, M. Sabir, and A. C. Bovik, “A statistical evaluation of recent full reference image quality assessment algorithms,” IEEE Transactions on Image Processing, vol. 15, no. 11, pp. 3440-3451, Nov. 2006. Article(CroosRef Link)

[22] C. Spearman, “The proof and measurement of association between two things," American Journal of Psychology, vol. 15, no. 1, pp. 72-101, Jan. 1904. Article(CroosRef Link)

[23] M. G. Kendall, “A new measure of rank correlation,” Biometrika, vol. 30, pp. 81-89, 1938.

[24] N. Damera-Venkata, T.D. Kite, W.S. Geisler, B.L. Evans, and A.C. Bovik, "Image quality assessment based on degradation model,” IEEE Transactions on Image Processing, vol. 9, no. 4, pp.636-650, 2000. Article(CroosRef Link)

[25] H.R. Sheikh, A.C. Bovik, and G. de Veciana, “An information fidelity criterion for image quality assessment using natural scene statistics,” IEEE Transactions on Image Processing, vol. 14, no. 12, pp. 2117-2128, 2005. Article(CroosRef Link)

[26] D.M. Chandler and S.S. Hemami, "VSNR: A wavelet-based visual signal-to-noise-ratio for natural images,” IEEE Transactions on Image Processing, vol. 16, pp.2284-2298, 2007. Article(CroosRef Link)

[27] L. Zhang, L. Zhang, and X. Mou, "RFSIM: a feature based image quality assessment metric using Riesz transforms," in Proc. of IEEE International Conference on Image Processing (ICIP), pp. 321-324, Sep. 2010. Article(CroosRef Link)

[28] H. R. Sheikh and A. C. Bovik, "Image information and visual quality," IEEE Transactions on Image Processing, vol. 15, no. 2, pp. 430-444, Feb. 2007. Article(CroosRef Link)

[29] E.C. Larson and D.M. Chandler, "Most apparent distortion: full-reference image quality assessment and the role of strategy,” Journal of Electronic Imaging, vol. 19, no. 1, pp. 1-21, 2010. Article(CroosRef Link)



Jee-Yong Lee received the B.S. degree in Department of Electrical and Computer Engineering from Ajou University, Suwon, Korea, in 2013. He is currently on the M.S. course in the embedded computing and systems lab. (ECSL) of the same department. His research interests include image processing and pattern recognition.

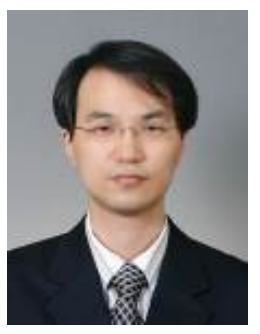

Young-Jin Kim received B.S. and M.S. degrees in electrical engineering and the Ph.D. degree in computer science and engineering from Seoul National University, Seoul, Korea, in 1997, 1999, and 2008, respectively. From 1999 to 2003, he was with the Electronics and Telecommunications Research Institute (ETRI), Daejoen, Korea. He was an assistant professor in the Department of Computer Science and Engineering, SunMoon University, Asan, Korea from 2008 to 2011. He is currently an associate professor in the Department of Electrical and Computer Engineering, Ajou University, Suwon, Korea. His research interests include embedded systems and software, display systems and image processing, storage systems, and low-power technology. 